\title{
Chromospheric Activity of Late-type Giants and Supergiants: Reappearance of Dynamo Activity in the Interior due to the Spin-up of the Core in Evolution
}

\author{
Yutaka Uchida and M. K. V. Bappu* \\ Tokyo Astronomical Observatory, University of Tokyo, Mitaka, Tokyo, 181 Japan
}

Received 1982 March 24; accepted 1982 July 5

\begin{abstract}
The renewal of chromospheric activity in red giants and supergiants is interpreted in terms of the reappearance of dynamo activity in the interior due to the spin-up of the core caused by its contraction in the course of evolution from the main sequence to the giant stage. A region of very high rotational shear (differential rotation) develops between the core, which spins up by a large factor through the drastic contraction, and the envelope, which spins down in contrast by virtue of expansion. Mechanisms of angular momentum transfer may operate to smear this large shear, and bring the inner part of the envelope into sheared rotation. A convective layer, on the other hand, develops in the envelope from the surface inwards, when the envelope expands and the temperature is lowered. A dynamo layer, or a layer in which the sheared rotation coexists with the convection (the presence of a remnant magnetic field being postulated), will thus reappear in the inner part of the envelope when the envelope-convection reaches down and invades the layer of sheared rotation. Surface chromospheric activity due to the magnetic field is thus renewed when the regenerated magnetic field is brought up to the surface by the envelope-convection. These phenomena occur as the star evolves into the giant stage and hence explain the observed characteristic of gradual revival of chromospheric activity from the subgiant to the giant stage.

Key words: stellar evolution-chromospheric activity-dynamo activity -red giants
\end{abstract} on 1982 August 19 


\section{Introduction}

$\mathrm{Ca}$ II $\mathrm{H}$ - and $\mathrm{K}$-line emission is well known to be a good indicator of the chromospheric magnetic activity in the case of the Sun (Thomas and Athay 1961; Bappu and Sivaraman 1971; Bray and Loughhead 1974; Skumanich, Smythe and Frazier 1975). The Ca II $\mathrm{K}$-line (Wilson and Bappu 1957; Wilson 1976), and more recently, resonance lines like $\mathrm{Mg}$ II $\mathrm{h}$ and $\mathrm{k}$ lines in the ultraviolet observed from space (Basri and Linsky 1979; Weiler and Oegerle 1979) have been extensively studied to investigate stellar chromospheres (Linsky 1980).

Two aspects of chromospheric behaviour have been the focus of much aftention. These are the relationship between $\mathrm{K}$-line widths and absolute magnitude (Wilson and Bappu 1957), and the age dependence of K-line emission intensities for stars on the main sequence (Wilson and Skumanich 1964; Skumanich 1972). The principal contributor to the integrated calcium emission spectrum that enables the Sun to follow the Wilson-Bappu relation is the bright fine mottle of size 1-2 arcsec (Bappu and Sivaraman 1971). There is little difference between the emission profile of the single bright fine mottle and the integrated spectrum of the Sun (Bappu and Sivaraman 1977). This is true at least for the quiet Sun; the localized enhancement that comes from active regions gives rise to a 'temporal' component that arises from the rotational modulation if the Sun is viewed as a star. The emission intensity has a linear dependence on surface magnetic field strengths to measurable low values in the case of the Sun (Frazier 1972), and there is enough circumstantial evidence available for the association of magnetic fields with the bright fine mottle (Bappu 1981). The decrease of chromospheric emission with age, experienced by stars on the main sequence, can well be considered to be due to a decrease in the magnetic field whose dissipation enhances chromospheric heating. Middelkoop and Zwaan (1981) have plotted Wilson's eye-estimates against $B-V$ values and find that the $G$ giants show a large spread in $\mathrm{H}$ and $\mathrm{K}$ emission; those giants that have broadened spectral lines tend to produce enhanced emission and hence are indicative of dynamo efficiency that is correlated with the rotation characteristic.

Bappu (1981) has shown recently from the distribution on the H-R diagram of latetype stars that show $\mathrm{K}$-line emission that, if traced back in time along the evolutionary track, the low-mass red giants-which today have chromospheric intensities that are strong and easily measurable - must have had periods in the late phase of their mainsequence stage during which they would have had no detectable chromospheric line emission. In a recent communication (Bappu 1982) he has used normalized Ca II chromospheric fluxes to show that it is necessary to postulate a renewal of magnetic activity in the atmosphere of the evolving star. Such renewal during the giant stage is, however, not expected from the simple application of the dynamo picture considered for main-sequence stars. The dynamo action in stars, which is an intensification of the pre-existing magnetic field by the cooperation of the sheared rotation and the convection (e.g. Parker 1955; Yoshimura 1975; Stix 1976), is expected to be strong in the envelope of young main-sequence stars-right after the Hayashi phase-due to the coexistence of strong convection and (sheared) rotation. Such dynamo action, however, decays through the long life in the main-sequence stage along with the decay of the stellar rotation, and the decay of the stellar rotation is more severe in going over to the giant stage due to the expansion and mass loss (Mestel 1972).

In the present paper, we propose an interpretation for the above-mentioned 
unexpected re-flourishing of the chromospheric magnetic activity in low-mass red giants in the following way. After the magnetic activity has ceased in these low-mass stars in the late phase of its main-sequence stage, the stars evolve into the giant stage in which the outer part of the star expands to form an extended envelope while the central part contracts to form a compact core because the central pressure cannot be maintained otherwise after the disappearance of the heat source at the centre (Schwarzschild 1958; Iben 1967a). If the star has been rotating at all, the core will spin up by a large factor due to the angular momentum conservation in the contraction whereas the envelope will spin down in the expansion for the same reason, and a large gradient in the angular velocity developes in the boundary region. Mechanisms of the angular-momentum transfer, such as the rotational instability causing turbulent viscosity (Goldreich and Schubert 1967; Fricke 1967) or the magnetic torquing (Lüst and Schlüter 1955; Mestel 1972), will then be set in operation and the core drags the inner part of the envelope into sheared rotation. The envelope, on the other hand, becomes convective starting from outside as the temperature is lowered due to the expansion in the course of evolution (Iben 1967a), and after some time the convection zone reaches down and invades the region which has been set into sheared rotation by the drag. A dynamo layer will thus be established and restarts the process of regeneration of the magnetic field. The magnetic field may be carried out to the surface by the envelope convection itself, but there exists another possibility that the magnetic bubble produced by the ballooning instability (Parker 1966) in the overlywound-up field in the sheared rotation directly emerges ( $c f$. Layzer, Rosner and Doyle 1980). According to the time-lag between the start of the evolution of a star on the evolutionary track to the giant stage, and the spinning-up of the core and deepening of the envelope convection, the chromospheric activity in red giants will be rekindled and will flourish some time after the star evolves into the giant stage, explaining the observation.

We first summarize the observational basis of the argument in Section 2. The spin-up of the core in evolution and the mechanisms of the angular momentum transfer caused by the large difference of the rotation of the core and the envelope are discussed in Sections 3 and 4, respectively. The renewal of the dynamo action and the reappearance of the magnetic field to the stellar surface is discussed in Section 5. Overall discussion is given in Section 6.

\section{The observational basis: a brief review}

Wilson's catalogue (1976) is a plentiful data source of K-emission intensities in latetype main-sequence stars, giants and supergiants. Stars in this catalogue are general galactic field stars and are given eye-estimates of chromospheric emission intensity on a scale of 1-5 (5 is the most intense). Ideally, we should have available normalized flux values of the emission to eliminate all effects of background variation caused by spectral type and luminosity differences. Very few of these, however, are available at the present time, and hence we use the eye-estimates that form a homogeneous set, for indicating post-main-sequence behaviour. Since, for all these stars the K-emission line widths provide absolute magnitudes, it is possible to plot all the stars on an $M_{v}$, $B-V$ plane. Fig. 1 shows such diagrams for different chromospheric intensity values. We have superposed on each of these diagrams the colour-magnitude loci of stars of 


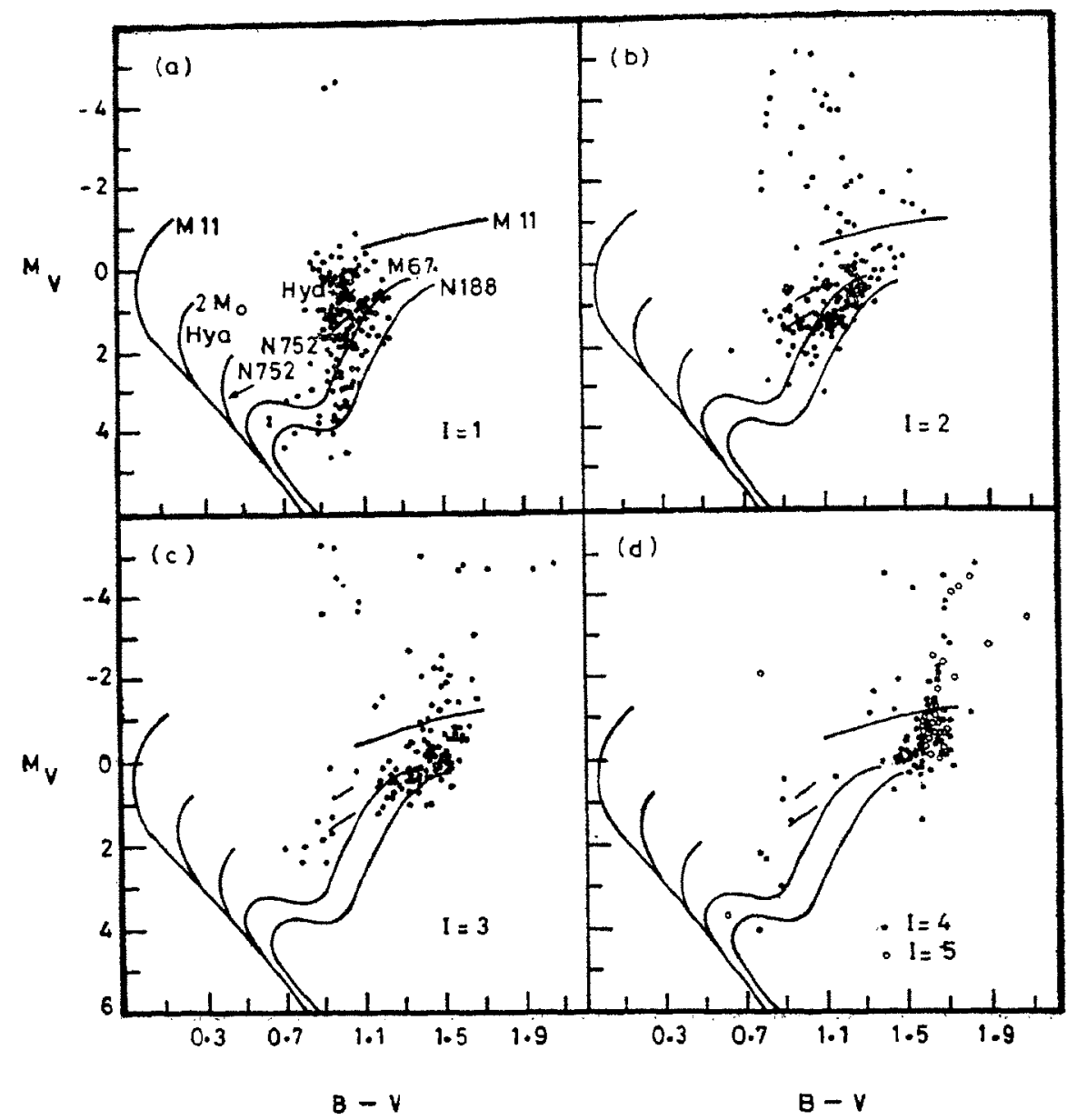

Figure 1. K-line-width absolute magnitude plotted against $B-V$ for different $K$-emission eyeestimates for stars of the general galactic field.

M 11, the Hyades, NGC 752, M 67 and NGC 188. The brightest stars in the Hyades have a turn-off point consistent with the Iben track of a $2 M_{\odot}$ star. Hence we use these locations to indicate the ages of the stars involved in the study.

There are several salient features in this diagram that are of interest. The higher chromospheric intensities display a migration to brighter absolute magnitudes and redder colours. Part of this shift can be ea, ily explained by the increase in apparent emission intensity as one assesses it against the weaker continuous background of later spectral types. To minimize its contribution we can make qualitative comparisons at any $B-V$ value. If we confine our attention to the narrow colour interval, $B-V$ $=0.9$ to 1.0 , and examine the vertical spread of the points, we find that we have an appreciable density of points that cover the absolute magnitude interval from about $M_{0}=-0.5$ to +4.0 for stars with intensity $I=1$, the number in this colour band decreasing as one goes to higher chromospheric intensity levels. The two extremes of luminosity available in this colour band in Fig. 1a are the giants younger than those in the Hyades and the subgiants comparable in age to those in M 67 or NGC 188. A 
low value of chromospheric emission for the luminous giants is consistent with their age if one assumes that the decay of emission has a rate independent of stage of evolution. In other words, these stars had chromospheres that-according to the Skumanich (1972) estimate of initial values - would be much greater, while still on the main sequence, than the present value of the Sun. On the other hand those stars located between the isochrones of the two very old galactic clusters must certainly have lost all traces of chromospheric emission before they have left the main sequence; that they are detectable at intensity level 1 on $10-\AA \mathrm{mm}^{-1}$ dispersion spectra is suggestive of a considerable revival of the chromospheric emission in the first stages of post-mainsequence evolution. By examining the star distribution in adjacent colour bands, we also note the steady enhancement of intensity, especially in the subgiant to giant phases of evolution.

This revival of chromospheric emission becomes apparent in our plot of the ratio of chromospheric flux in the $\mathrm{Ca}$ In lines to the bolometric luminosity for stars where such information is available in the literature (Fig. 2). The Ca II and $\mathrm{Mg}$ II radiative loss rates are taken from Linsky et al. (1979) as well as Basri and Linsky (1979). Dwarfs, giants and supergiants are identified separately. The numbers on each point in the diagrams of both lines represent the Wilson eye-estimates of $\mathrm{Ca}$ II emission intensity. The insufficiency of systematically obtained observational data is an obvious characteristic with ground-based CaII observations-the sparser of the two. We notice that the values of flux are shared by main-sequence objects, the supergiants and some of the giants. As plotted therein, on a two dimensional diagram, there is no systematic dependence of the ratio of the chromospheric flux and the total luminosity on the luminosity class. We note, however, that in general, stars of higher luminosity emit proportionally stronger $\mathrm{K}$ - or $\mathrm{k}$-line emission at any effective temperature. In each djagram, the curve defining a limit of chromospheric emission (LCE) beyond which a normal star of luminosity class I, III or V can seldom be located, is drawn in Bappu (1982). The drawing of this curve at low effective temperatures is aided by the eye-estimates of $\mathrm{K}$ emission; we know from experience that brighter cases than those marked in the diagram are seldom seen amongst the large number of stars in the solar neighbourhood. Its position is particularly uncertain at the higher effective temperatures corresponding to the later classes of spectral type $F$. The fact that we can specify an empirically located LCE implies that, in the overall energy balance scheme, the chromospheric contribution has an upper limit that is seldom exceeded and which depends on the effective temperature. In postulating any mechanism for the generation of chromospheric emission, the magnitudes to be sought for in the energy generation are limited by the LCE. A peak in the chromospheric-flux distribution normalized to the bolometric flux has been shown some years ago by Blanco et al. (1974). The LCE is now used to introduce (Bappu 1982) the display of the age parameter, the evaluation of which is to be done by a measure of the deviation from it of the flux values of any star. Consider the vertical distribution of points at $5600 \mathrm{~K}$ that passes through the location of the quiet Sun. With intensity 4 near the LCE and the Sun located far from it, one can interpret the K-emission decay seen on the main sequence (Skumanich 1972) as measurable in terms of the deviation from the LCE. For $T_{\mathrm{e}}=4300 \mathrm{~K}$ we have a variety of objects located over a wide range of chromospheric flux in both sections of Fig. 2. The main-sequence star in the Ca II diagram with a large deviation from the LCE is an old object despite its high eyeestimate value of emission intensity, and typifies the effect of background contrast.

\section{A. -5}



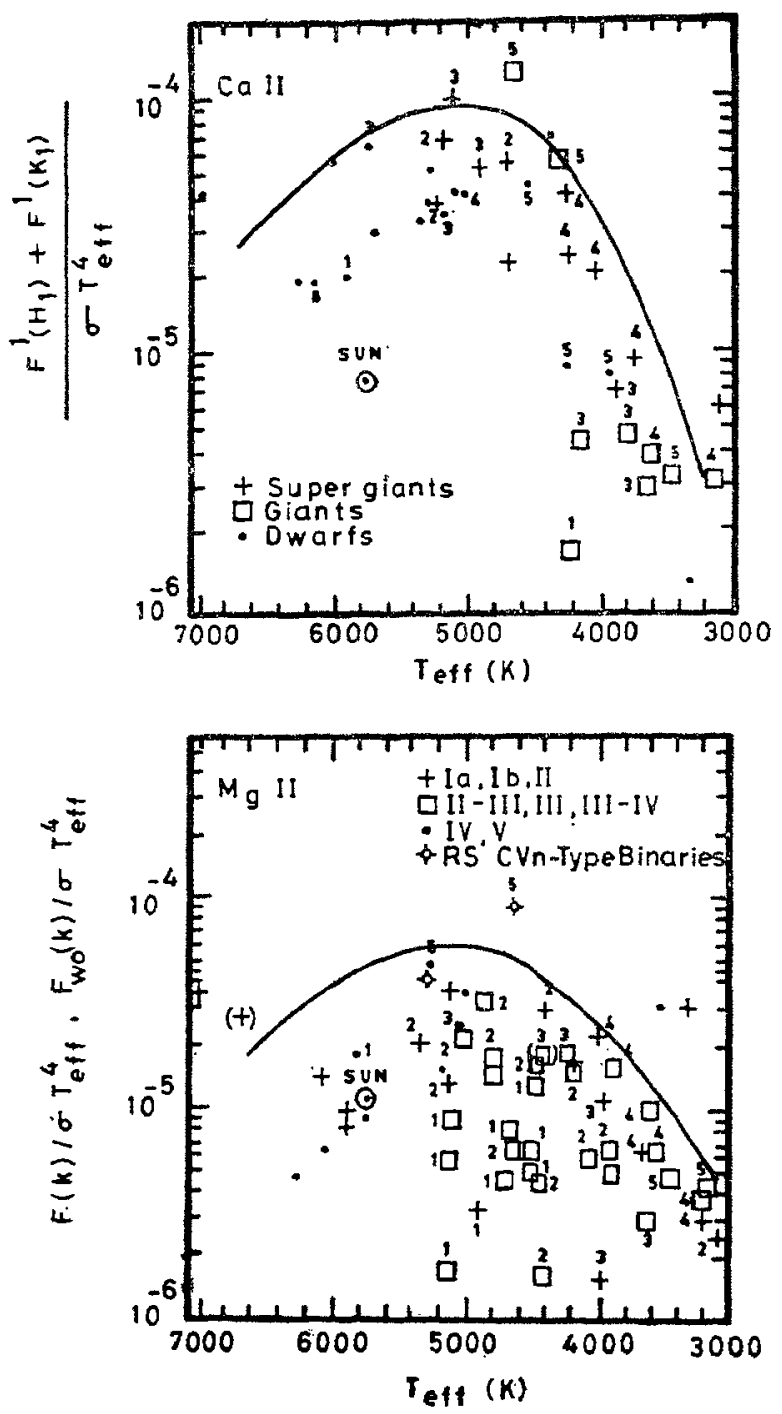

Figure 2. The ratio of chromospheric flux to bolometric luminosity for different values of $T_{\mathrm{e}}$ for the $\mathrm{Ca}$ in and $\mathrm{Mg}$ in lines (see text).

There are supergiants near the LCE and even a single giant that is an RS CVn object with enhanced contribution from plages. Notice the lone K1 IV object that is so far from the LCE and which, on the basis of the arguments presented earlier, will acquire more chromospheric emission as it evolves further. It would then drift higher up and to the right, closer to the LCE, as several other giants on the diagram have presumably done. A main-sequence star moves away from the LCE with age while a star evolving into a giant attempts to get closer to it. With the sparse data we now have, it will not be possible to indicate whether in this second phase of chromospheric renewal the star can reach the LCE. The supergiants have the best of both contributions and hence hover aro und the LCE. 
Their present chromospheric intensities may benefit also from the original surface magnetic fields that have not had time to decay in addition to the new contributions acquired by virtue of the changes in the post-main-sequence evolution. Observations of $\mathrm{K}$ emission in old galactic clusters like $\mathrm{M} 67$ assume a role of importance in providing evidence for such conjectures of chromospheric revival. Such observations, difficult as they are likely to be, are nevertheless within the scope of today's technology.

\section{Evolution of the internal state of rotation-spin-up of the core}

As mentioned in Section 1, it is well known that a compact core is formed by contraction during the course of evolution of a star into the giant stage. Our proposal here is based on the change in the distribution of the angular velocity inside a star (if it is rotating) due to such a change in the distribution of mass in the course of evolution.

In order first to estimate the upper limit of the rotational velocity of the spun-up core, we assume for the time being that there exists no mechanism of angular-momentum exchange between thin elementary rings [located at $\left(r_{0}, \theta_{0}\right)$ in the spherical coordinate whose origin is at the centre of the star and the axis coincides with the axis of the rotation], and that each ring expands or contracts to its new $(r, \theta)$ in the course of evolution without interacting with one another. The conservation of angular momentum $J$ of a thin elementary ring,

$\frac{d J}{d t}=0$

implies that

$J=r^{2} \sin ^{2} \theta \Omega \Delta M=$ const $\equiv r_{0}^{2} \sin ^{2} \theta_{0} \Omega_{0} \Delta M_{0}$

where $\Delta M$ is the mass of the ring, $\Omega$ is the angular velocity, and the subscript 0 denotes those quantities before the particular part of the evolution under consideration takes place. Since $\Delta M$ is conserved and $\theta$ is constant in the radial contraction or expansion, we have,

$\frac{\Omega}{\Omega_{0}}=\left(\frac{r}{r_{0}}\right)^{-2}$

We can calculate the change in the angular velocity of the ring by using this relation with the calculated model stellar evolution which gives the change with time of $r(m, t)$, where $m \equiv M_{r} / M_{*}$ is the mass-fraction from the centre. Fig. 3 shows the change in the distribution of the mass before and after the evolution from Iben's model calculations for the evolution of a $1 M_{\odot}$ star (Iben 1967b). Using similar graphs also for $1.25 M_{\odot}$ and $1.5 M_{\odot}$ stars from Iben (1967b), we can obtain the expected ratios of the angular velocity before and after the evolution (normalized 


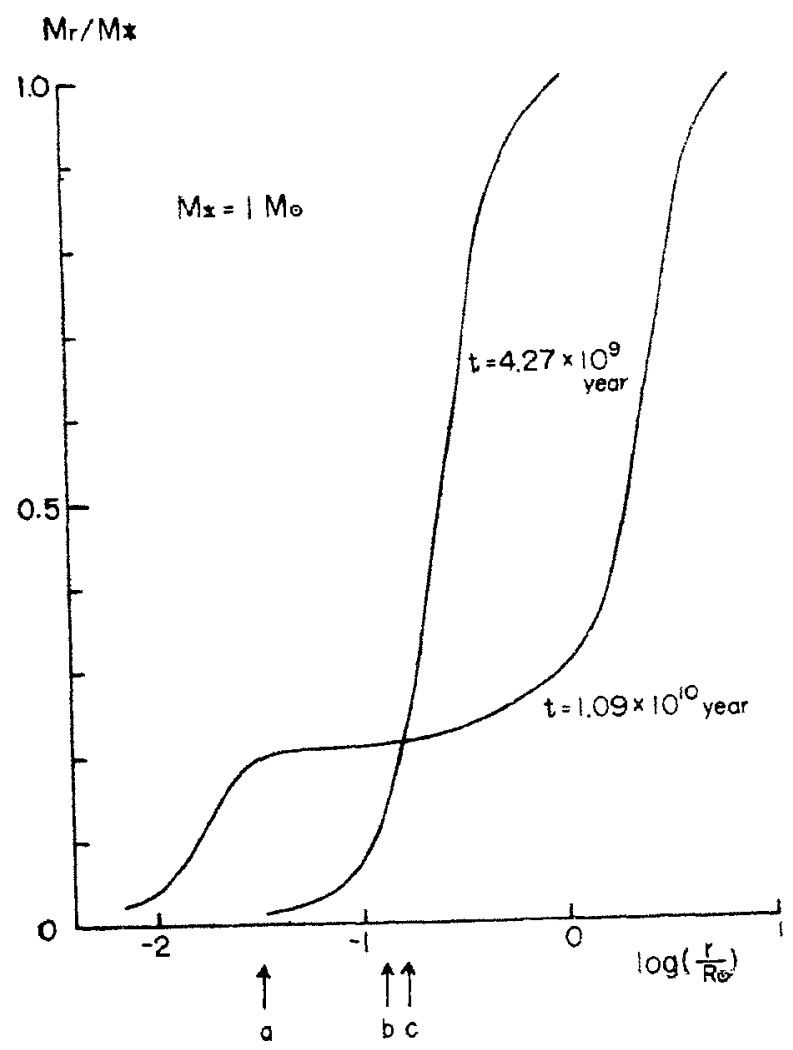

Figure 3. $\left(r / R_{\odot}\right)-\left(M_{r} / M_{*}\right)$ diagram for $1 M_{\odot}$ star at $t=4.27 \times 10^{9} \mathrm{yr}$ and $1.09 \times 10^{10} \mathrm{yr}$ (from Iben 1967b). This shows that the part $r>r_{s s}$ expands roughly by one order of magnitude while $r<r$ contracts by about the same factor during the time interval, where $r_{s s l}$ is the radius of the stand-still layer. The edge of the core, the bottom of the envelope convection, and the stand-still layer are indicated by arrows $\mathrm{a}, \mathrm{b}$ and $\mathrm{c}$, respectively, for the curve of $t=1.09 \times 10^{10} \mathrm{yr}$.

to the corresponding values before the evolution) in these stars* (Fig. 4). It is seen that the core spins up by a large factor. Kippenhahn (1963) has mentioned this aspect in a different context of a possible modification of stellar evolution. A more elaborate discussion on these lines is seen in Endal and Sofia (1976). Also, a large shear in the angular velocity is seen to build up in the region surrounding the core in all these models. The region with large gradient in $\Omega$ is the region of sheared rotation, and the top of it may be defined by a radius which fulfils the equation $\Omega(m)=\Omega_{0}(m)$. This particular layer neither expands nor contracts (stand-still layer) and keeps its original angular velocity. The layer of sheared rotation appears due to the structural change in the star, and the latter becomes appreciable some time after the star evolves away from the main sequence. Fig. 5 shows the edge of the core and the stand-still layer in terms of the mass-fraction, and Fig. 6 shows the epoch at which the structural change

* Observed stellar properties such as rotational velocity or surface convection differ for stars with different masses along the main sequence. The ratio $\Omega / \Omega_{0}$, however, depends only on the change in the distribution of mass inside the star. The presence of convection near the surface, or the presence of internal differential rotation do not matter. The presence of intemal rotation will affect the detailed value of $\Omega$ through $\Omega_{0}$, but our conclusion of faster-rotating core will not be affected unless the profile of initial rotational velocity has a sharp decrease toward the core. 


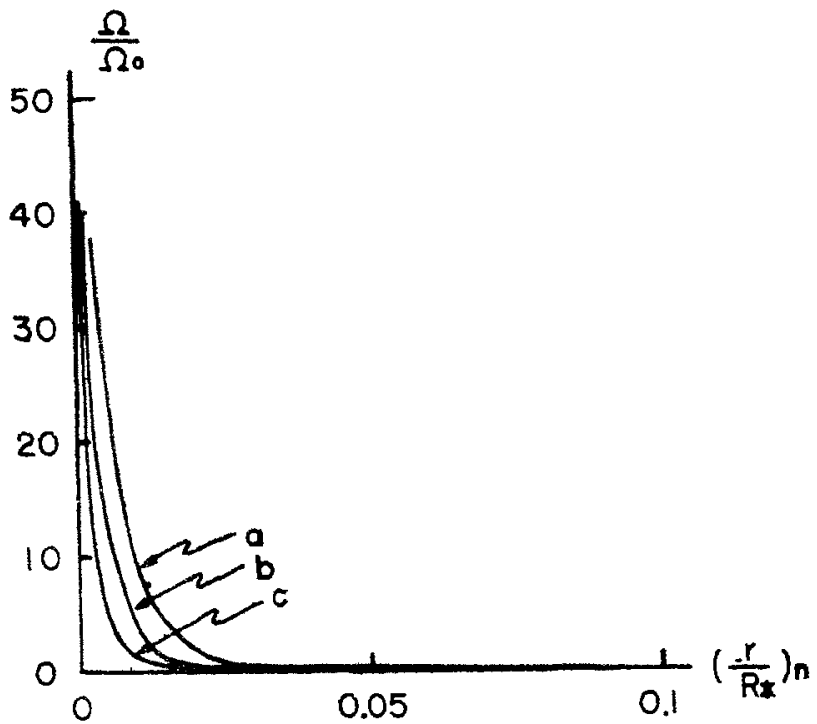

Figure 4. $\left(r / R_{*}\right)-\left(\Omega / \Omega_{0}\right)$ curves $\mathrm{a}, \mathrm{b}$ and $\mathrm{c}$ of the spin-up calculated for $M=1 M_{\odot}, 1 \cdot 25 M_{\odot}$ and $1.5 M_{\odot}$ model stars of Iben, respectively.

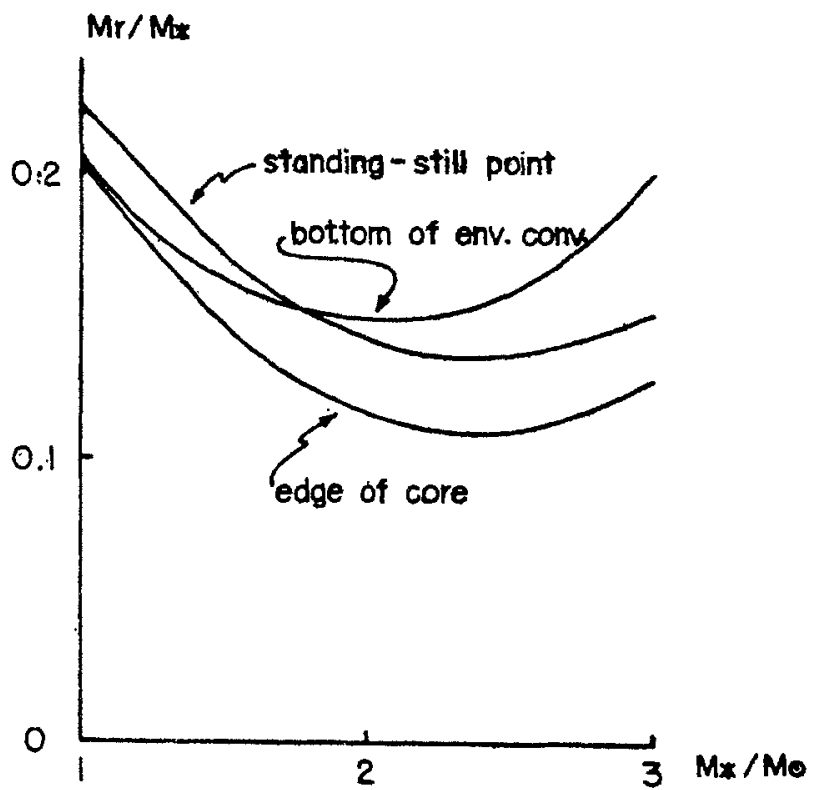

Figure 5. Mass-fractions of the edge of the core, of the stand-still layer, and of the bottom of the envelope of the model stars as functions of stellar mass, deduced from the calculations of Iben $(1967 a, b, c)$.

becomes appreciable; these are all derived from Iben's models for $1 M_{\odot}, 1.25 M_{\odot}$, $1.5 M_{\odot}, 2.25 M_{\odot}$ and $3 M_{\odot}$ stars (Iben 1965, 1967b, 1967c).

On the other hand, the convection develops from the surface inwards due to the cooling of the envelope by expansion, and finally covers most of the envelope. The greatest depth which the bottom of the envelope convection reaches and the time 


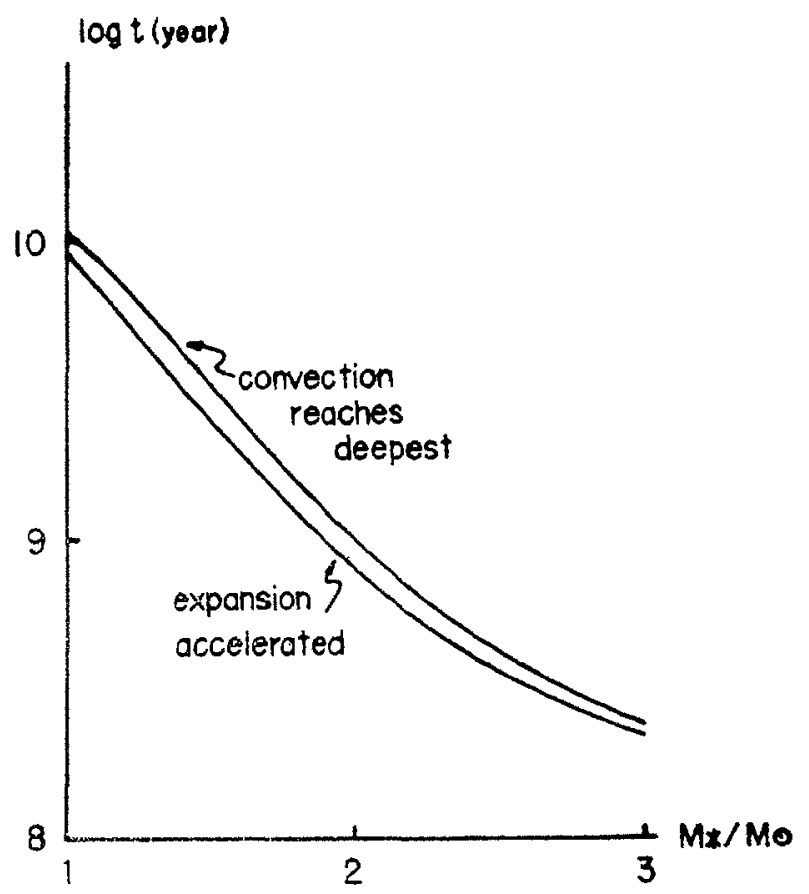

Figure 6. Epochs at which the change of the internal structure is accelerated in the course of evolution, and at which the bottom of the envelope-convection reaches its greatest depth, as functions of stellar mass (deduced from the calculations of Iben $1967 \mathrm{a}, \mathrm{b}, \mathrm{c}$ ).

taken to reach this state are also plotted in Figs 5 and 6, respectively, for each of ${ }^{\circ}$ these models. The radii of the edge of the core, of the stand-still layer, and of the bottom of the envelope convection are indicated in Fig. 3 for the case of $1 M_{\odot}$ star, as an example. It is seen from Fig. 6 that the bottom of the envelope convection is inside the region where the strong shear in the rotation exists. In the case of more massive stars, however, the bottom of the envelope convection lies outside the region of sheared rotation, but not very far away. There exist mechanisms of angular-momentum transfer which tend to relax the large gradient in $\alpha$, or by which the fast-rotating core drags the inner part of the convective envelope and brings it into a sheared rotation. A 'dynamo-layer' which has both sheared rotation and convection in it (the presence of a remnant magnetic field being postulated) will thus appear in these heavier stars also, while it is strengthened in the case of the $1 M_{\odot}$ star. In Section 4, we will seek mechanisms which allow such angular-momentum transfer. Finally, Fig. 7 shows the locations on the $\mathrm{H}-\mathrm{R}$ diagram at which the structural changes are accelerated and the bottom of the envelope convection reaches its greatest depth, respectively, on Iben's model evolutionary tracks. These coincide with the renewed appearance of chromospheric emission after the evolution of the stars to the giant stage (Bappu 1981).

The problem of 'the rapidly rotating core of red giants and the angular momentum transfer to the envelope' reminds us of Dicke's (1964) proposal of the 'rapidly rotating core of the Sun' and the controversial debate on its spin-down (Howard, Moore and Spiegel 1967; Goldreich and Schubert 1967; Fricke 1967; Sakurai 1975). This problem and ours are directly related, but the parameters involved are very 


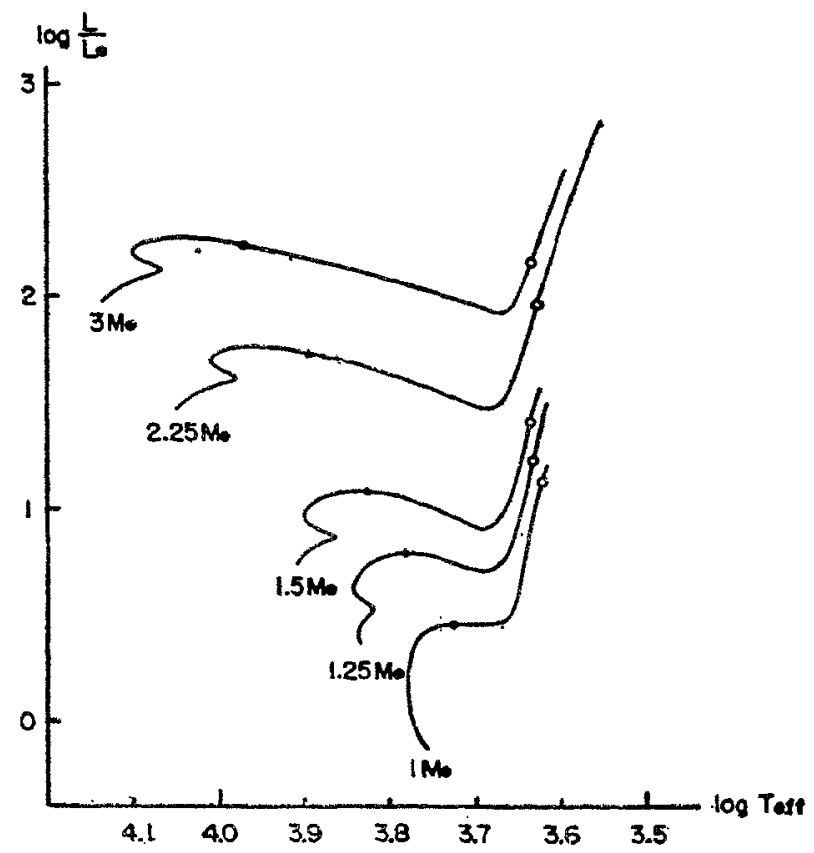

Figure 7. Locations along Iben's (1967a) evolutionary tracks on the H-R diagram of the epochs of acceleration of structural changes in evolution (dots) and of envelope-convection reaching the greatest depth (open circles).

different. For example, the radius and the central density of the core in a giant of $R_{*} \simeq(10-20) R_{\odot}$ in our case are of the order of $0.03 R_{\odot} \simeq(0.003-0.0015) R_{*}$ and $10^{5} \mathrm{~g}$ $\mathrm{cm}^{-3}$, respectively, while in Dicke's solar case $\left(R_{*}=1 R_{\odot}\right)$ these are $0.53 R_{\odot}=0.53 R^{*}$ and $10^{2} \mathrm{~g} \mathrm{~cm}^{-3}$, respectively. Therefore, most of the mechanisms of the spin-down that argued against Dicke's hypothesis by relying upon the interaction through the interface of the core and the envelope, become ineffective in our case of red giants due to the very small surface area of the core.

\section{Mechanisms of angular momentum transfer from the core to the inner part of the envelope}

There are some mechanisms of angular momentum transfer which may still be efficient with this small surface area of the rotating core even though the efficiency may be low. This decreased efficiency, however, is in a sense favourable, because we do not want a mechanism which is so efficient that it smears out all the differential rotation and makes the star rotate quasi-rigidly, thus destroying the merit of the differentially rotating interior. The total angular momentum of the core, if redistributed evenly, ensures only a rather slow rotation even for stars initially rotating rapidly as seen in Section 6 .

\subsection{Goldreich-Schubert-Fricke Mechanism}

One possibility of a suitable mechanism is the Goldreich-Schubert-Fricke mechanism 
which uses the eddy viscosity of the turbulence caused by the instability of the sheared rotation when either one or both of the conditions

$\frac{\partial\left(\widetilde{\omega}^{2} \Omega\right)}{\partial \widetilde{\omega}}>0$

and

$\frac{\partial \Omega}{\partial z}=0$

where $\widetilde{\omega} \equiv r \sin \theta$, are violated (Fricke 1967). $\widetilde{\omega}$ and $z$ are coordinates in a cylindrical coordinate system $(\hat{\omega}, \phi, z)$ with the axis of rotation coinciding with the $z$-axis. These criteria are violated in our case. According to Kippenhahn and Thomas (1981), the eddy viscosity $\eta_{\mathrm{t}}$ due to the destabilized axisymmetric torus is given as $\eta_{\mathrm{t}}=\rho v_{\mathrm{t}} d$, where $\rho$ is the density, $d$ is the cross-sectional size of the torus which is assumed to dissolve as it moves a distance of its own size, and $v_{t}$ is the velocity of the element estimated by

$v_{t} \simeq \frac{H_{p}}{H_{\Omega}} \frac{2 \chi}{\nabla_{\mathrm{ad}}-\nabla} \frac{d}{\tau^{*}}$

Here $H_{p} \equiv \mathscr{R} T \widetilde{\omega}^{2} / G M_{r}$ is the scaleheight, $H_{\Omega} \equiv\left\{-\partial \ln \left(\tilde{\omega}^{2} \Omega\right) / \partial \widetilde{\omega}\right\}^{-1}$ is the scale length of variation of $\widetilde{\omega}^{2} \Omega, \chi \equiv \widetilde{\omega}^{3} \Omega^{2} / G M_{r}$ is the ratio of centrifugal to gravitational forces, $\nabla \equiv \partial \ln T / \partial \ln p$, and $\tau^{*}$ is the timescale of the thermal adjustment, $\tau^{*}=3 C_{p} \kappa \rho^{2} \zeta d^{2} / 8 a c T^{3}$, in which $C_{p}$ is the specific heat at constant pressure, $\kappa$ is the absorption coefficient per mass, $a$ is the Stefan-Boltzmann constant, $c$ is the velocity of light, and $\zeta$ is a constant factor of order unity. $\nabla_{\mathrm{ad}}-\nabla$ and $\tau^{*}$ come into the expression since the element moves up because of the temperature stratification with the heat exchange by radiation. $\eta_{\mathrm{t}}$ is thus given by,

$\eta_{t} \simeq \frac{16}{3} \frac{\chi}{\nabla_{\mathrm{ad}}-\nabla} \frac{H_{p}}{H_{\Omega}} \frac{a c T^{3}}{\zeta C_{p} \kappa \rho}$

The angular momentum transport by the turbulent viscosity caused by this mechanism may occur diffusively as

$\frac{\partial}{\partial t}\left(\rho \tilde{\omega}^{2} \underline{\Omega}\right)=\frac{1}{\tilde{\omega}} \frac{\partial}{\partial \widetilde{\omega}}\left(\eta_{t} \tilde{\omega}^{3} \frac{\partial \Omega}{\partial \widetilde{\omega}}\right)$

and the timescale for the diffusion may roughly be estimated as

$\tau_{\mathrm{GSF}} \simeq \frac{\rho(\Delta \widetilde{\omega})^{2}}{\eta_{\mathrm{t}}} \simeq \frac{\zeta C_{p} \kappa \rho^{2}}{16 a c T^{3}} \frac{\nabla_{\mathrm{ad}}-\nabla}{\chi} \frac{H_{\Omega}}{H_{P}}(\Delta \widetilde{\omega})^{2}$ 
where $\Delta \widetilde{\omega}$ is the width of the region over which the transport takes place. This may be compared with Dicke's case as

${ }_{\mathrm{GSF}} \simeq\left(\frac{\rho}{\rho_{\mathrm{D}}}\right)^{2}\left(\frac{T}{T_{\mathrm{D}}}\right)^{-1 / 2}\left(\frac{M_{\mathrm{r}}}{M_{r \mathrm{D}}}\right)^{2}\left(\frac{\widetilde{\omega}_{t}}{\widetilde{\omega}_{t \mathrm{D}}}\right)^{-5}\left(\frac{\Delta \widetilde{\omega}}{\Delta \widetilde{\omega}_{\mathrm{D}}}\right)^{2}\left(\frac{\Omega}{\Omega_{\mathrm{D}}}\right)^{2}\left(\frac{H_{\Omega}}{H_{\Omega \mathrm{D}}}\right)\left(\frac{\left(\nabla_{\mathrm{ad}}-\nabla\right)}{\left(\nabla_{\mathrm{ad}}-\nabla\right)_{\mathrm{D}}}\right) \tau_{\mathrm{GSF}} \mathrm{D}$

Subscript $D$ refers to the values in the boundary region in Dicke's case. Roughly estimating ratios $\rho / \rho_{\mathrm{D}}$ etc. from the model, $\tau_{\mathrm{GSF}}$ in our case turns out to be $10^{3} \sim 5$ times of $\tau_{\mathrm{GSF}, \mathrm{D}}$. The estimate of $\tau_{\mathrm{GSF}, \mathrm{D}}$ itself, however, was controversial as mentioned above, ranging from $10^{5} \mathrm{yr}$ (Goldreich and Schubert 1967) to the Kelvin-Helmholz timescale (Kippenhahn 1969) or longer due to the non-linear suppression of the mode (James and Kahn 1970, detailed discussion may be found in Fricke and Kippenhahn 1972).

If the value of $\tau_{\mathrm{GSF}, \mathrm{D}}$ falls on the smaller side in the range of the estimates, the Goldreich-Schubert-Fricke mechanism may serve in thickening the 'tail' in the distribution of the rotational shear. The mechanism is self-breeding (i.e. the newly produced region of sheared rotation will in turn produce turbulence which then serves in transporting angular momentum outwards) and therefore it may work even if the size of the core is small. However, the action of it may not be powerful enough to cover the entire star, since the new supply of the angular momentum from the dense core to the 'tail' cannot be too effective due to the compactness of the core, and also the expansion of the turbulent region may be suppressed by nonlinear effects (James and Kahn 1970).

\subsection{Magnetic Torquing}

Another possibility is magnetic torquing (e.g. Lüst and Schlüter 1955). We assume that certain magnetic field remains inside the star from the earlier phase of the mainsequence stage in which the dynamo activity existed, but weakened later on. The weakening of the dynamo activity may be due to the decay of the differential rotation which in turn may be due to the damping of the rotation itself in the later phase of the main-sequence stage. Since the diffusion time of the once-generated magnetic field in the star, $\tau_{\mathrm{d}}=4 \pi \sigma R_{*}^{2} / c^{2}$, is rather long (of the order of $10^{10} \mathrm{yr}$ for Spitzer conductivity), the magnetic field may be retained even after the dynamo action has ceased.

When the redistribution of the mass takes place in the course of evolution, a redistribution of the magnetic field may also be caused due to the frozen-in character of the magnetic field. The magnetic field in the outer part is expanded along with the expansion of the envelope, and that of the inner part is contracted with the contracting core. What is to be noted is the behaviour of the magnetic field in the region of sheared rotation (Section 3 ). The field lines having one end in the core and the other in the envelope will be wound up quickly and tightly due to the large difference in the rotational velocity of the core and the envelope.

Angular momentum transport from the rapidly-rotating core to the envelope may be due to the tension of the thus-wound-up field exerted on the inner-most part of the envelope in which the extention of the field lines is imbedded. The magnetic 
torque exerted over the inner surface of the low-latitude part of the shell which lies immediately outside of the region of sheared rotation may be written as

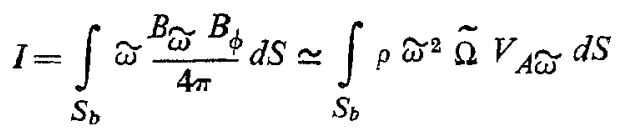

where $S_{b}$ is the low-latitude part of the inner surface of the shell immediately outside of the shearing region [for example, $(\pi / 2) \widetilde{\omega}_{b}^{2}$ ] $V_{A \widetilde{\omega}}=B_{\widetilde{\omega}} /(4 \pi \rho)^{\frac{1}{2}}, B_{\phi}^{2} / 8 \pi \simeq \rho \widetilde{\omega}^{2}{\tilde{\Omega^{2}}}^{2} / 2$, and $\tilde{\Omega}$ is the angular velocity of the shell relative to the region of the wound-up magnetic field which is considered to be almost corotating with the core. The adopted picture is that the field is stretched by the sheared rotation to such a degree in which the weaker side of the anchoring ground (inner edge of the shell immediately outside of the shearing region) can barely stand. Since $I$ is roughly: $(\pi / 2) \rho_{b} \widetilde{\omega}_{b}^{4} \tilde{\Omega}_{b}$ $V_{A \widetilde{\omega}_{b}}$, and $J_{b}=\widetilde{\omega}_{b}^{2} \tilde{\Omega}_{b} \Delta M_{b}=(\pi / 2) \rho_{b} \widetilde{\omega}_{b}^{4} \tilde{\Omega}_{b} \Delta \widetilde{\omega}$, the equation of the spin-up of the shell, $d J / d t=I$, may be written as

$\frac{\pi}{2} \rho_{b} \widetilde{\omega}_{b}^{4} \Delta \widetilde{\omega} \frac{d \tilde{\Omega}_{b}}{d t} \simeq \frac{\pi}{2} \rho_{b} \widetilde{\omega}_{b}^{4} \tilde{\Omega}_{b} V_{A \widetilde{\omega}_{b}}$

and therefore

$\frac{d \ln \tilde{\Omega}_{b}}{d t} \simeq \frac{V_{A \widetilde{\omega}_{b}}}{\Delta \widetilde{\omega}}$

The timescale of the magnetic spin-up is therefore

$\tau_{\mathrm{msu}} \simeq \frac{\Delta \widetilde{\omega}}{V_{A \widetilde{\omega}}}$

coinciding with the expression given by Mouschovias (1981) for the magnetic braking in the star formation. $\tau_{\mathrm{msu}}$ is rather short, of the order of $10^{10} \mathrm{~s}$ for $\Delta \widetilde{\omega} \sim 10^{11} \mathrm{~cm}$, even with a low estimate of the order of $10^{2} \mathrm{G}$ for the $\widetilde{\omega}$-component of the remnant magnetic field at the bottom of the envelope. The winding-up of the field and the drag due to the tension of this field take place almost immediately compared with the Kelvin-Helmholz timescale. How, then, is it possible that the effect of this does not dominate and bring the entire star into corotation? There are many factors which can prevent such a dominance. For example, since the small spherical core is not an ideal spindle which can hold a large amount of wound-up field on it, the field will slip off poleward and the wound-up region will not continue to grow beyond a certain radius which is a small fraction of the stellar radius. This radius, however, may be larger than the radius to which the bottom of the convection zone comes down, since these radii are rather close from each other and a dynamo layer will be formed.

It is also possible that the wound-up field loaded with gravitated mass is unstable for the perturbation as in the ballooning instability argued by Parker (1966) for the 
formation of the cloud in the galactic plane. Once a part of the field is raised, the mass will slide down to the lower part, and the evacuated part of the field-line will float up fulther. This popped up part will serve as a paddle around the rotator and enhances the angular momentum transfer to the surrounding envelope. The timescale of the rise will again be a short dynamical timescale but again the range in radius for the angular momentum transfer is restricted in favour of our requirement, since the field strength in the bubble decreases as $\left[\left(\widetilde{\omega}-\widetilde{\omega}_{b}\right) / b\right]^{-3}$, where $b$ is the horizontal scale of the perturbation on the sphere at the bottom of the envelope, and is not large. The capability of this magnetic paddle in giving angular momentum to the surrounding mass will decrease quickly as the bubble rises.

One or other of the mechanisms of angular momentum transfer discussed thus far, therefore, seem to be appropriate for our requirement since they are effective in ranges short compared with the radius of the star, $R$, but long enough to produce a region of sheared rotation in the convective envelope, the bottom of which comes down close to the edge of the core (Section 2) and forms the so-called dynamo layer.

\section{Dynamo action and the emergence of the magnetic field to the stellar surface}

Dynamo mechanism developed for the Sun refers to the regeneration process of the magnetic field in the differentially-rotating convective atmosphere. The differential rotation stretches the field and produces the toroidal component (Parker 1955; Babcock 1961), and the convection with the effect of Coriolis force serves as the backfeeder from the toroidal to the poloidal field (Parker 1955). Our situation described above in which the inner part of the convective envelope is dragged into a sheared rotation through the coupling with the rapidly rotating core, just provides us with a dynamo layer.

The equation describing this regenerative process of the magnetic field may be written in the form,

$$
\begin{aligned}
& \frac{\partial A}{\partial t}=a_{A A} A+a_{A B} B \\
& \frac{\partial B}{\partial t}=a_{B B} B+a_{B A} A
\end{aligned}
$$

where $A$ is the $\varphi$-component of the vector potential and $B$ is the averaged toroidal field intensity, and represent poloidal and toroidal field, respectively. The expressions of coefficients differ from one model to another, depending upon the flow patterns and velocity amplitude considered in the model.

For example, in Yoshimura's model (1972), which is one of the most successful models introducing the notion of the global convection, these are,

$$
\begin{aligned}
& a_{A A} \equiv D_{s}+D_{G A}, \\
& { }_{B B} \equiv D_{s}+D_{G B},
\end{aligned}
$$


$a_{A B} \equiv R_{G A}$,

$a_{B A} \equiv R_{D R}+R_{G B}$,

where

$D_{G A} \equiv \frac{U^{2}}{2 r_{s}^{2}\left|\omega^{(0)}\right|} L_{A}(\mu, z)$,

$D_{G B} \equiv \frac{U^{2}}{2 r_{s}^{2}\left|\omega^{(0)}\right|} L_{B}(\mu, z)$,

$R_{G A} \equiv \frac{U^{2}}{2 r_{s}^{2}\left|\omega^{(0)}\right|} \frac{D}{\pi} \frac{\Omega_{e}}{\left|\omega^{(0)}\right|} L_{A}(\mu, z)$,

$R_{G B} \equiv \frac{U^{2}}{2 r_{s}^{2}\left|\omega^{(0)}\right|} \frac{\pi}{D} \frac{\Omega_{e}}{\left|\omega^{(0)}\right|} L_{B}(\mu, z)$,

$D_{s} \equiv \frac{\eta_{\mathrm{m}}}{r_{s}^{2}} L_{s}(\mu, z)$,

$R_{D R} \equiv \frac{a_{1} \Omega_{e}}{D} L_{D R}(\mu, z)$,

in which $L_{A}(\mu, z)$ etc. are operators inherent to the assumed flow-patterns, $\omega^{(0)}$ is the angular frequency of the dynamo cycle, $\Omega_{e}$ and $a_{1}$ are the angular velocity and the coefficient of its $\left(1-\mu^{2}\right)$ dependence at the inner radius of the dynamo layer, respectively; $r_{s}$ is the inner radius, $D$ is the thickness of the dynamo layer, $U$ is the velocity amplitude of the convection, and $\eta_{\mathrm{m}}$ is the magnetic diffusivity. Coefficients $D_{G A}, D_{G B}$ and $D_{s}$ represent dissipative effects by convection and by magnetic diffusivity respectively, and $R_{G A}, R_{G B}$ and $R_{D R}$ represent regenerative effects by convection and by differential rotation, respectively. It is to be noted that the convection contributes to the dissipation of $A$ and $B$ as well as to the regeneration of $A$ from $B$ while the differen tial rotation contributes to the generation of $B$ from $A$ and to the regeneration of $A$ from $B$.

From the discussion of Sections 3 and 4, we here assume, as a set of rough parameters, that $\Omega_{e}$ is greater by a factor $10, U$ is smaller by a factor 10 , respectively, than those in the solar case, while other parameters are of the same order as those of the solar case. Then, modifying the values in Yoshimura's (1975) estimates according to their dependence on $\Omega_{e}$ and $U$, we have the estimates for the coefficients as,

$$
\begin{aligned}
& \left|D_{G A}\right| \sim\left|D_{G B}\right| \sim 10^{-11} \mathrm{~s}^{-1},\left|D_{s}\right| \sim 10^{-9} \mathrm{~s}^{-1} \\
& \left|R_{G A}\right| \sim 5 \mathrm{~cm} \mathrm{~s}^{-1},\left|R_{G B}\right| \sim 4 \times 10^{-20} \mathrm{~cm}^{-1} \mathrm{~s}^{-1} \\
& \text { and } \\
& \left|R_{D R}\right| \sim 10^{-15} \mathrm{~cm}^{-1} \mathrm{~s}^{-1}
\end{aligned}
$$


It is seen from this that the production of the toroidal field from the poloidal field is enhanced by one order of magnitude mainly due to the effect of the high differential rotation. The regeneration of the poloidal from the toroidal is smaller by one order of magnitude due to the enhancing effect by large $\Omega_{e}$ and the reducing effect by small $U$. Dissipation effect by convection is weaker by two orders of magnitude due to the assumed smaller velocity amplitude of the convection at the bottom of convection zone.

A very rough measure of the regeneration may be given by the ratio of $B_{\mathbf{p}} / B_{t}$ when $A$ reaches an extremum $(\partial A / \partial t \simeq 0$ in Equation 15) which is

$$
\frac{B_{p}}{B_{t}} \simeq \frac{\frac{1}{r} \frac{\partial(r A)}{\partial r}}{B} \sim \frac{A}{r B} \sim \frac{R_{G A}}{r_{s} D_{s}} \sim 5 \times 10^{-2} .
$$

If $B_{t}$ is a fraction of the wound-up field which is stretched by the difference in the rotation between the core and the envelope, $B_{t} \simeq\left(4 \pi_{\rho_{b}}\right)^{1 / 2} \widetilde{\sim}_{b} \widetilde{\Omega}_{b} \sim 10^{6} \mathrm{G}, B_{p}$ can attain high enough a value as the regenerated source.

Marked differences between our case and the solar case are as follows. For the mechanism of the maintenance of the differential rotation in the solar case, some special mechanism of angular momentum transport to the region of higher angular velocity at low latitudes has been considered in order to explain the equatorial acceleration on the surface (Busse 1970; Durney 1970). In contrast, in our case, the differential rotation is sustained simply by the gradual transfer of the angular momentum from the spun-up core to the inner-most part of the envelope.

Also, the dynamo layer in our case is located very deep in the envelope around $R \sim 0.1 R_{*}$, while in the solar case it is in the outer convection zone of $R>0.85 R_{*}$. The magnetic field at the upper boundary of the dynamo layer is directly observable in the solar case, but the corresponding layer is deep-lying and the magnetic field released from this deep-lying dynamo layer is carried out to the stellar surface diffusively by convection. This may make the emerging magnetic flux diffuse in an extended area in the case of giants. The detection of stellar rotation may then be possible either from the flux variation of chromospheric lines (Wilson 1978), or from the variation of the total light (e.g. Eaton and Hall 1979 for RS CVn case).

\section{Discussion}

First, it is interesting to ask whether our picture for low-mass giants is also relevant for the case of supergiants. These objects, which have evolved from massive stars, had greater rotational velocities and probably stronger fossil magnetic fields than those of low-mass giants when they were on the main sequence. It therefore seems possible that the chromospheric activity of them may be either due to the diluted fossil field itself, or due to the field regenerated in the dynamo process by the differential rotation which survived the spin-down of the convective envelope.

We may, however, argue against these possibilities. Take as an example a B5type $\operatorname{star}\left(M_{*}=6 M_{\odot}, R_{*}=4 R_{\odot}, V \sin i \lesssim 160 \mathrm{~km} \mathrm{~s}^{-1}\right.$ and, say, $\left.B_{\text {surf }} \sim 3 \times 10^{3} \mathrm{G}\right)$. In the course of evolution, it will expand to $10^{2} R_{\odot}$; the fossil magnetic field of even 
$3 \times 10^{3} \mathrm{G}$ on the average on the main sequence will be diluted to $3 \times 10^{3} \times(1 / 25)^{2}$ $\sim 5 \mathrm{G}$, and the angular velocity, which was $20 \Omega_{\odot}$, will drop to $20 \Omega_{\odot} \times(1 / 25)^{2} \sim$ $0.032 \Omega_{\odot}$. We thus note in the case of supergiants that the effect of rapid initial rotation tends to be cancelled by the larger spin-down ratio due to the larger ratio of radii in the expansion. These estimates show that the diluted fossil field and the spun-down angular velocity of the envelope are too small to do much. Non-fossil, or non-potential field character is indeed required in order to explain the magnetic activity including the heating of the corona (e.g. Uchida 1980).

In contrast to these, the process similar to the one discussed in the present paper will inevitably take place also in supergiants. Spin-up of the core is more pronounced than in the case of low-mass giants due to the larger ratio of radii in the contraction. Also, the regenerated field in supergiants will be stronger than in the case of giants, since the density in the rapidly rotating core and in the surroundings, the anchorground for stretching the field in the region of sheared rotation, is higher and can windup the toroidal field stronger. If diffused to a similar field strength over the larger surface area of supergiants, this may explain the observation of Basri and Linsky (1979) and Weiler and Oegerle (1979) that supergiants emit one to two orders of magnitude more energy in the chromospheric lines than the giants.

It should be mentioned that the detailed values of the resulting magnetic field, for example, depend not only on the mass but also on the distribution of the initial magnetic field and initial rotation in the interior. This may explain the scatter in the observed intensities of $\mathrm{Ca}$ II or $\mathrm{Mg}$ II lines for stars with the same mass since initial magnetic field and initial rotation are the factors which also depend on the circumstantial condition at the star formation, rather than solely on the mass of the star.

Finally, we may make a comment on the relation of the rotation of a star to its magnetic activity (Ayres and Linsky 1980; Walter 1981; Vaiana 1980). It has usually been considered by applying the standard picture of the dynamo action that, among late-type stars with surface convection, those with higher rotational velocity may be regenerating stronger magnetic field. This picture may well be true in the case of young late-type dwarfs, or in the case of synchronously-rotating binaries like RS CVn type. In the case of single giants and supergiants, however, the discussion above suggests that it may not be the surface rotational velocity inherited from the rotation in the main sequence that is responsible for the dynamo action. How, then, is the correlation of the magnetic activity with the surface rotational velocity interpreted in our picture? A possible answer to this question is that, contrary to the usual view, the latter may be due to the angular momentum transported from the interior, e.g. by the magnetic field effect, and thus the excess angular velocity over the value expected from the angular momentum conservation of individual shells goes parallel to the appearance of the internally-produced magnetic field to the surface. Indeed some of the chromosphere-active giants or supergiants seem to have larger rotational velocities than what is explicable by the spin-down according to the angular momentum conservation of the shell of the surface mass.

\section{Acknowledgements}

One of the authors (M. K. V. B.) acknowledges the support of his stay at the Tokyo Astronomical Observatory by the Japan Society for the Promotion of Sciences. He 
also thanks Professor Y. Kozai, the director, and the staff of the Observatory for their hospitality. The authors express their gratitude to Mrs. H. Suzuki and Mr. Y. Shiomi for their help in preparing the manuscript.

\section{References}

Ayres, T. R., Linsky, J. L. 1980, Astrophys. J., 241, 279.

Babcock, H. D. 1961, Astrophys. J., 133, 572.

Bappu, M. K. V. 1981, Bok Festschrift, Ed. R. E. White (in press).

Bappu, M. K. V. 1982, Curr. Sci., 51, 24.

Bappu, M. K. V., Sivaraman, K. R. 1971, Solar Phys., 17, 316.

Bappu, M. K. V., Sivaraman, K. R. 1977, Mon. Not. R. astr. Soc., 178, 279.

Basri, G. S., Linsky, J. L. 1979, Astrophys. J., 234, 1023.

Blanco, C., Catalano, S., Marilli, E., Rodono, M. 1974, Astr. Astrophys., 33, 257.

Bray, R. J., Loughhead, R. E. 1974, The Solar Chromosphere, Chapman and Hall, London.

Busse, F. H. 1970, Astrophys. J., 159, 629.

Dicke, R. H. 1964, Nature, 202, 432.

Durney, B. 1970, Astrophys. J., 161, 1115.

Eaton, J. A., Hall, D. S. 1979, Astrophys. J., 227, 907.

Endal, A. S., Sofia, S. 1976, Astrophys. $I ., 210,184$.

Frazier, E. N. 1972, Solar Phys., 24, 98.

Fricke, K. 1967, PhD Thesis, University of Göttingen.

Fricke, K. J., Kippenhahn, R. 1972, A. Rev. Astr. Astrophys., 10, 45.

Goldreich, P., Schubert, G. 1967, Astrophys. J., 150, 571.

Howard, L. N., Moore, D. W., Spiegel, E. A. 1967, Nature, 214, 1297.

Iben, I. Jr. 1965, Astrophys. J., 142, 1447.

Iben, I. Jr. 1967a, A. Rev. Astr. Astrophys., 5, 571.

Ibe., I. Jr. 1967b, Astrophys. J., 147, 624.

Iben, I. Jr. 1967c, Astrophys. J., 147, 650.

James, R. A., Kahn, F. D. 1970, Astr. Astrophys., 5, 232.

Kippenhahn, R. 1963, in Star Evolution, Ed. L. Gratton, Academic Press, New York, p. 330.

Kippenhahn, R. 1969, Astr. Astrophys., 2, 309.

Kippenhahn, R., Thomas, H. C. 1981, in Proc. IAU Symp. 93: Fundamental Problems in the Theory of Stellar Evolution, Eds D. Sugimoto, D. Q. Lamb and D. N. Schramm, D. Reldel, Dordrecht, p. 237.

Layzer, D., Rosner, R., Doyle, H. T. 1980, Centre for Astrophys. Preprint No. 1078.

Linsky, J. L. 1980, A. Rev. Astr. Astrophys., 18, 439.

Linsky, J. L., Worden, S. P., McClintock, W., Robertson, R. M. 1979, Astrophys. J.Suppl. Ser., . $41,47$.

Lüst, R., Schlüter, A. 1955, Z. Astrophys., 38, 190.

Mestel, L. 1972, in Stellar Evolution, Eds H.-Y. Chiu and A. Muriel, MIT Press, Cambridge, p. 643 .

Middelkoop, F., Zwaan, C. 1981, Astr. Astrophys., 101, 26.

Mouschovias, T. C. 1981, in Proc. IAU Symp. 93: Fundamental Problems in the Theory of Stellar Evolution, Eds D. Sugimoto, D. Q. Lamb and D. N. Schramm, D. Reidel, Dordrecht, p. 27.

Parker, E. N. 1955, Astrophys. J., 122, 293.

Parker, E. N. 1966, Astrophys. J., 145, 811.

Sakurai, T. 1975, Mon. Not. R. astr. Soc., 171, 35.

Schwarzschild, M. 1958, Stellar Structure and Evolution, Princeton University Press.

Skumanich, A. 1972, Astrophys. J., 171, 565.

Skumanich, A., Smythe, C., Frazier, E. N. 1975, Astrophys. J., 200, 747.

Stix, M. 1976, in Proc. IAU Symp. 71: Basic Mechanisms of Solar Activity, Eds V. Bumba and J. Kleczek, D. Reidel, Dordrecht, p. 367.

Thomas, R. N., Athay R. G. 1961, Physics of Solar Chromosphere, Interscience, New York. 
Uchida, Y. 1980, in Proc. Japan-France Seminar on Solar Physics, Eds S. F. Moriyama and J.-C. Henoux, Tokyo Astronomical Observatory, p. 83.

Vaiana, G. 1980, in ISAS Symp. on Space Astrophysies, Ed. S. Hayakawa, ISAS, p. 173.

Walter F. M. 1981, Astrophys. J., 245, 677.

Weiler, E. J., Oegerle, W. R. 1979, Astrophys. J. Suppl. Ser., 39, 537.

Wilson, O. C. 1976, Astrophys. J., 205, 823.

Wilson, O. C., Bappu, M. K. V. 1957, Astrophys. J., 125, 661.

Wilson, O. C., Skumanich, A. 1964, Astrophys. J., 140, 1401.

Yoshimura, H. 1972, Astrophys. J, 178, 863.

Yoshimura, H. 1975, Astrophys. J. Suppl. Ser., 29, 467. 